\title{
Erratum: Excitation and charge transfer in low-energy hydrogen-atom collisions with neutral atoms: Theory, comparisons, and application to Ca [Phys. Rev. A 93, 042705 (2016)]
}

\author{
Paul S. Barklem
}

(Received 30 May 2017; published 23 June 2017)

\author{
DOI: 10.1103/PhysRevA.95.069906
}

In making comparisons with other studies and codes, two errors have been found. The first concerns the approximate long-range $\mathrm{H}^{-}$spatial wave-function $\varphi_{\mathrm{LR}}$ as described in Eq. (13) and accompanying text. The stated values for $N=0.223106$ and $r_{0}=0.2355885$ a.u. give the correct long-range behavior for a nonantisymmetrized total ionic state wave function of the form $\Phi_{1}=\varphi_{1 s}\left(\overrightarrow{r_{1}}\right) \varphi_{\mathrm{LR}}\left(\overrightarrow{r_{2}}\right)$ as was used by Adelman and Herschbach in Ref. [15] of the original paper and Anstee in Ref. [20] of the original paper. However, for the case of a correctly antisymmetrized function $\Phi_{1}$ as used in the paper, the additional normalization factors mean $\varphi_{\mathrm{LR}}$ should be adjusted to obtain the correct long-range behavior of $\Phi_{1}$. Solving the relevant equations, it is found that the correct values required to give $\Phi_{1}$ correct long-range behavior while retaining correct normalization are instead $N=0.333672$ and $r_{0}=2.30986$ a.u. Thus, the $\mathrm{H}^{-}$wave function used was too small at long range by a factor of roughly $1 / 3$, leading to couplings underestimated by a similar factor. The second error is that a bug was found in the computer code to calculate the couplings for the semiempirical (SEMI-EMP) model, used for comparisons with the linear combinations of atomic orbital (LCAO) model. Both these errors caused the couplings to by chance be underestimated by roughly similar magnitude, and thus, since the two methods were in reasonable agreement, the two errors were obscured. When corrected, the first error propagates through all calculations and means that Figs. 3-8 are all changed; new figures are given here and captions are unchanged except
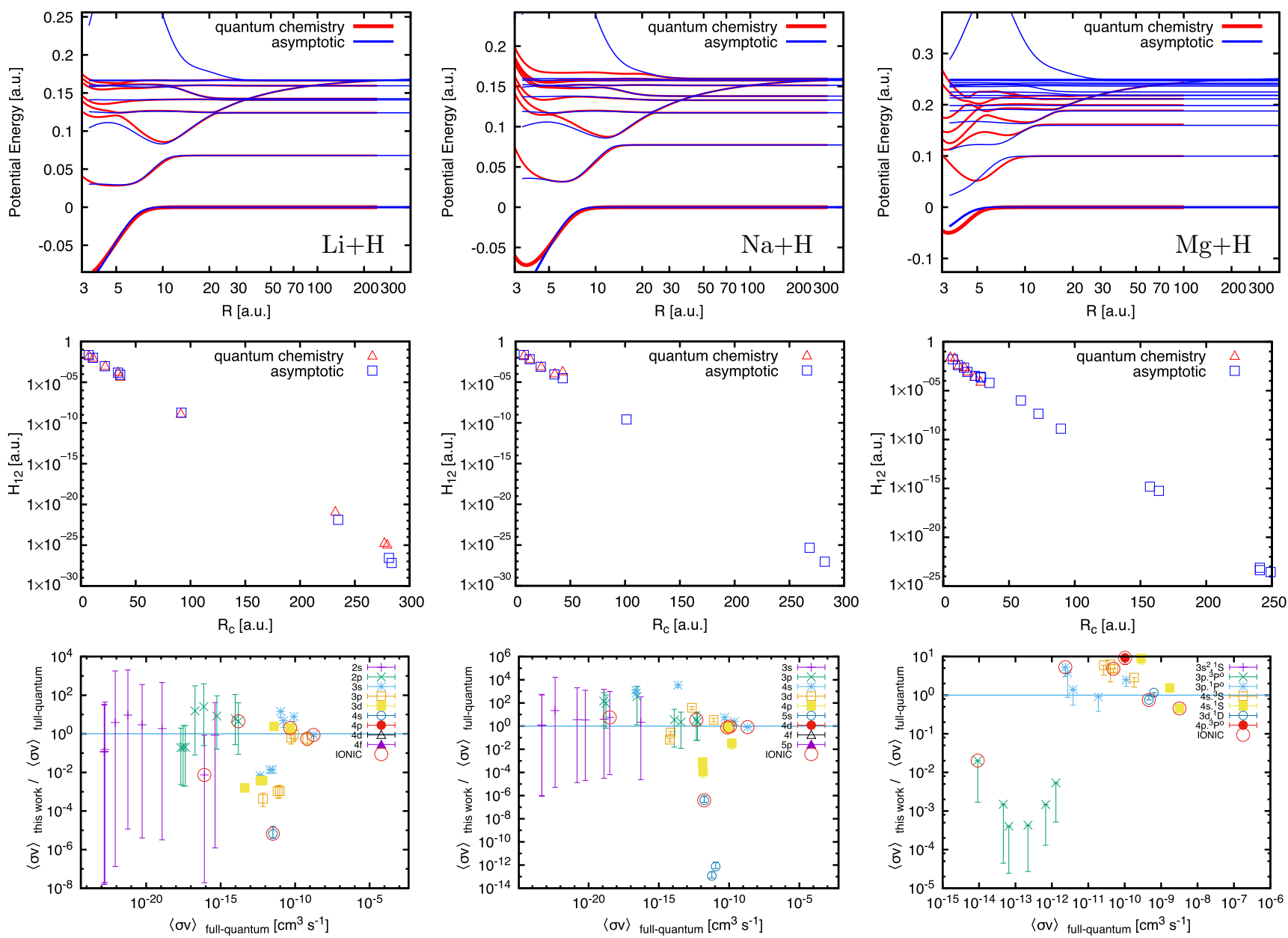

FIG. 3. Revised comparisons of calculations in the $\mathrm{LCAO}$ asymptotic model for $\mathrm{Li}+\mathrm{H}, \mathrm{Na}+\mathrm{H}$, and $\mathrm{Mg}+\mathrm{H}$ with results from full-quantum scattering calculations and the quantum chemistry data they are based on. Note, the crossing for $\mathrm{Mg}+\mathrm{H}$ involving $3 s^{2}{ }^{1} \mathrm{~S}$ now occurs at very short range $R<3$ a.u. in the diabatic model and cannot be treated with the asymptotic model. 

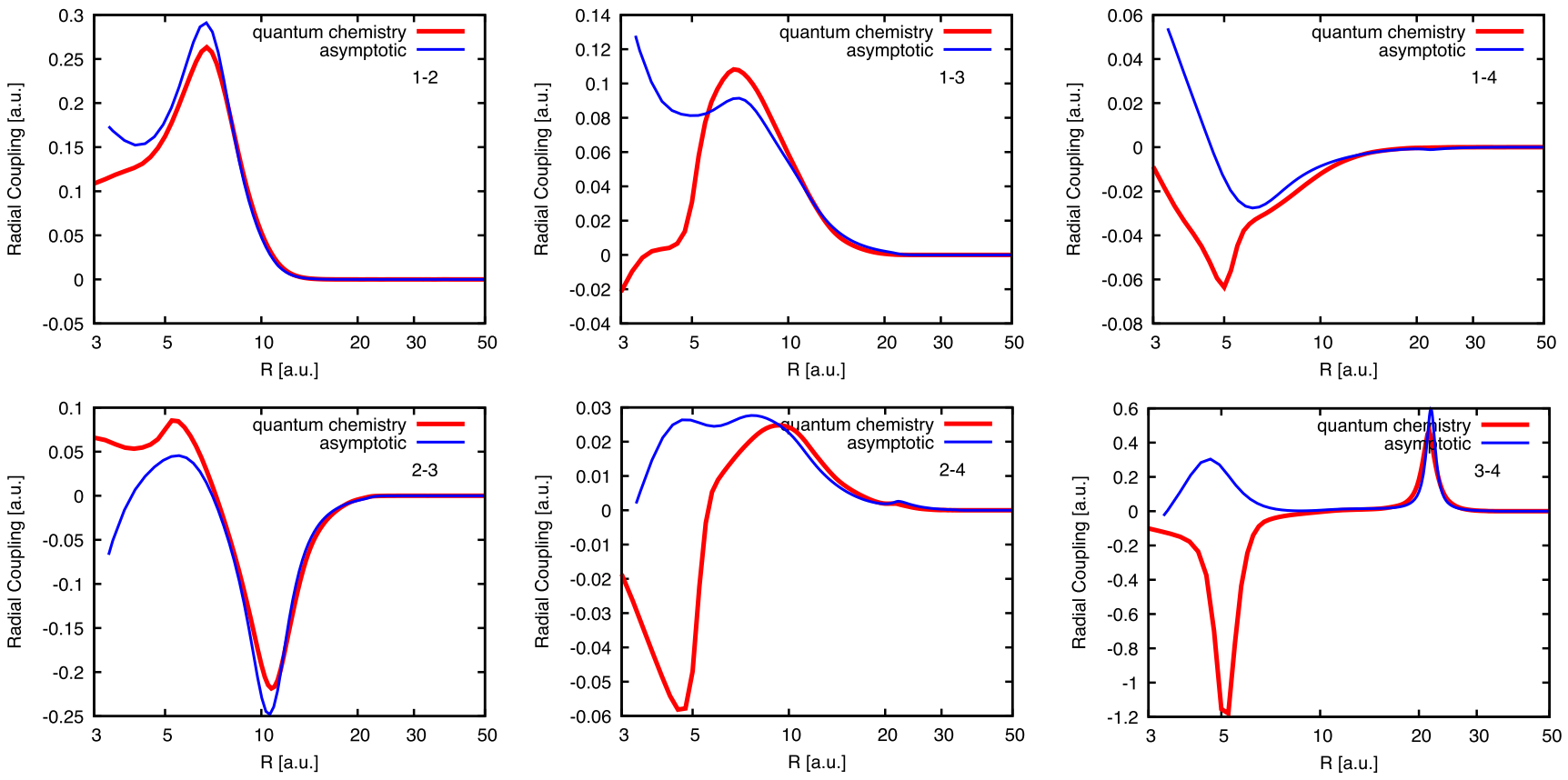

FIG. 4. Revised comparison of radial couplings for the $\mathrm{LiH}\left({ }^{1} \Sigma^{+}\right)$quasimolecule.

as noted. The comparisons with the full-quantum calculations generally are improved. In particular, the large discrepancies with the full-quantum results for small rates in $\mathrm{Li}+\mathrm{H}$ and $\mathrm{Na}+\mathrm{H}$ are reduced significantly, although those for $\mathrm{Mg}+\mathrm{H}$ are somewhat increased; see Fig. 3. Agreement of potential curves, couplings, and radial couplings is also generally improved; see Figs. 3 and 4. For the comparisons for different methods, with these corrections and improvements to the codes, it is now found that generally the LCAO, SEMI-EMP, and Landau-Herring-Janev (LH-J) models perform roughly equally well on average, all significantly better than Landau-Herring-Smirnov (LH-S). Note, fluctuation factors shown in figures are often smaller than in the original paper due to removal of the LH-S model, which often dominated these factors. The Supplemental Material with the paper has been updated with corrected data for $\mathrm{Ca}+\mathrm{H}$.
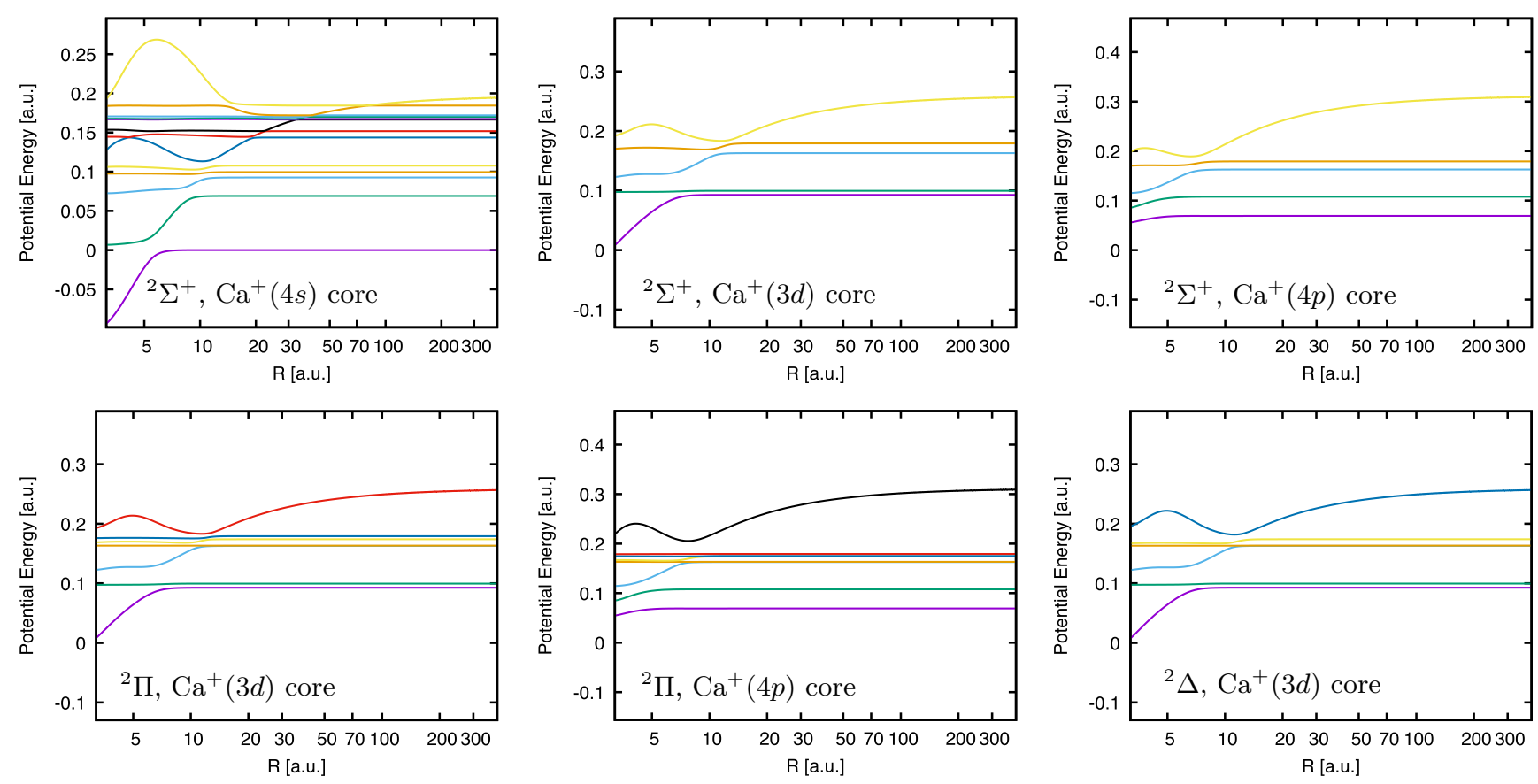

FIG. 5. Revised potentials energies for $\mathrm{Ca}+\mathrm{H}$. 

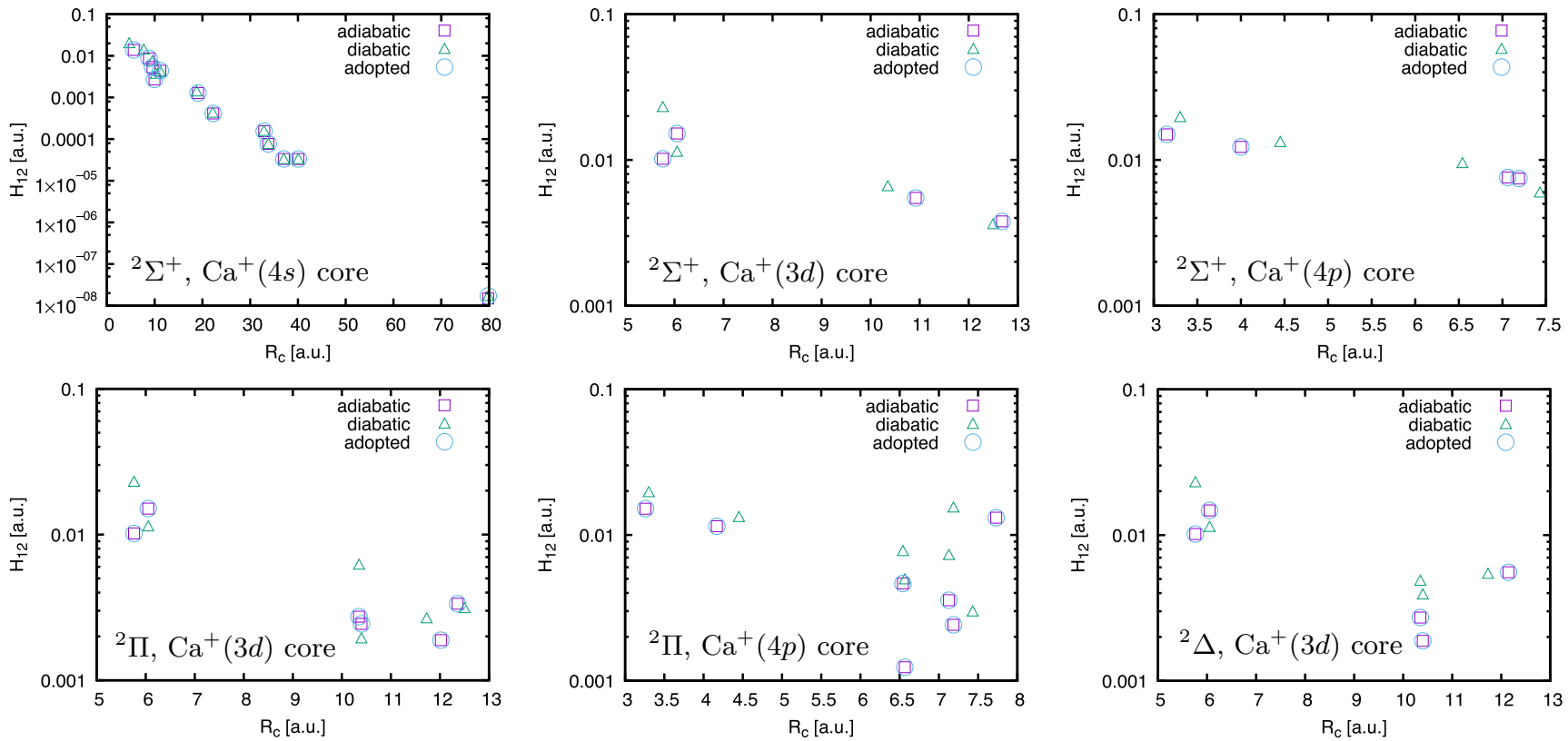

FIG. 6. Revised Landau-Zener parameters for $\mathrm{Ca}+\mathrm{H}$.

Finally, the opportunity is taken to correct a few minor typographical errors. In Eqs. (16) and (17), the 2 in the denominators should be inside the square root. In the line preceding (A1), the phrase "and $S_{0 L} \rightarrow 0$ " should be removed. In the last line of Eq. (A3), the factor 2 in the denominator should be removed. In Eq. (A8), $S_{01}$ has incorrectly been written instead of $S_{0 L}$.

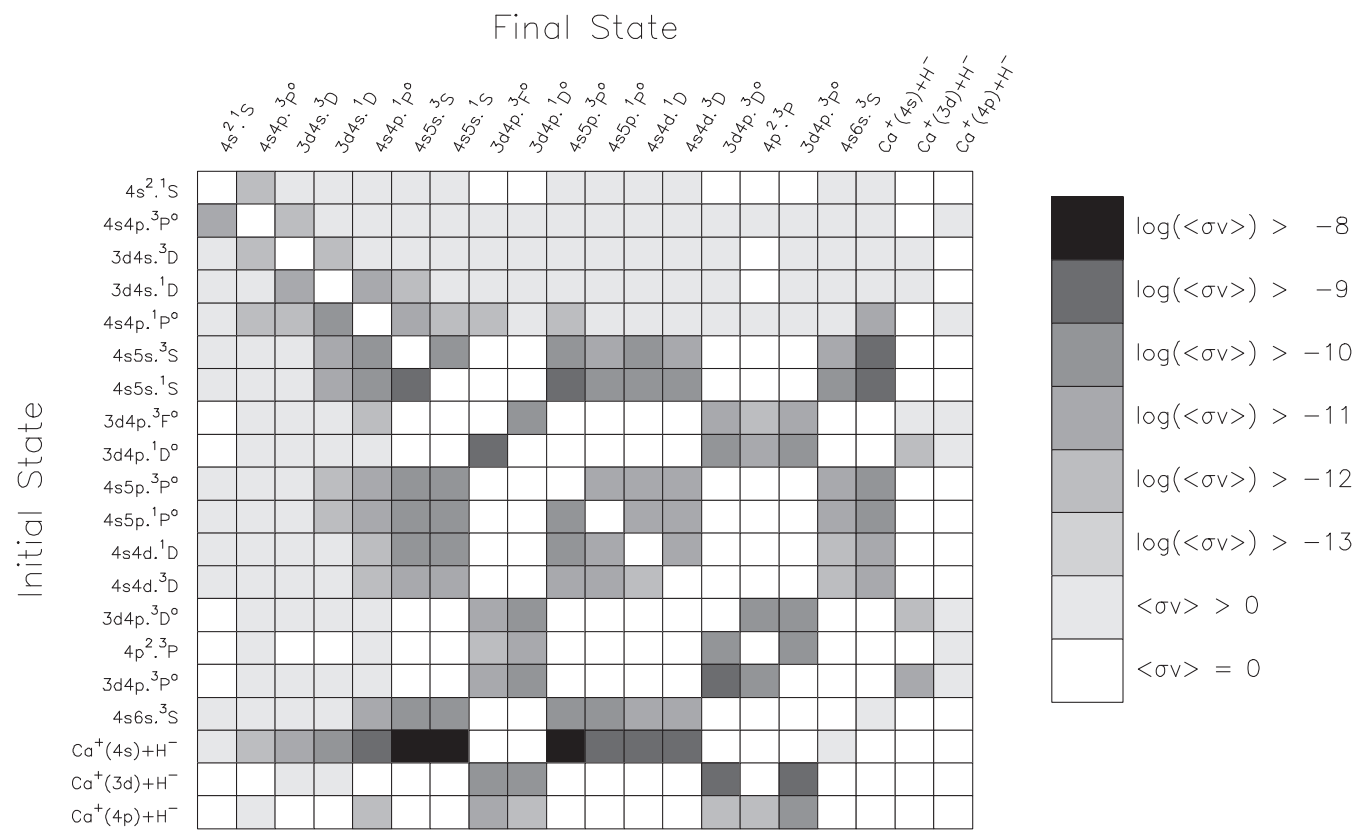

FIG. 7. Revised graphical representation of the rate coefficient matrix $\langle\sigma v\rangle\left(\right.$ in $\mathrm{cm}^{3} \mathrm{~s}^{-1}$ ) for inelastic $\mathrm{Ca}+\mathrm{H}$ and $\mathrm{Ca}^{+}+\mathrm{H}^{-}$collisions at temperature $T=6000 \mathrm{~K}$. 


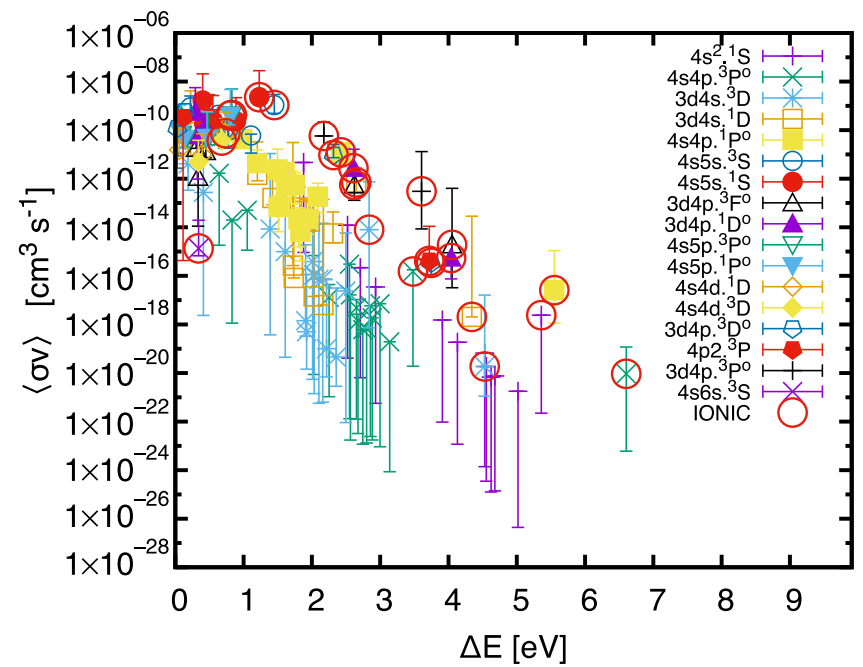

FIG. 8. Revised rate coefficients $\langle\sigma v\rangle$ for $\mathrm{Ca}+\mathrm{H}$ collision processes at $6000 \mathrm{~K}$, plotted against the asymptotic energy difference between initial and final molecular states $\Delta E$. 